\title{
Differentiability Properties of the Pressure in Lattice Systems
}

\author{
H. A. M. Daniëls and A. C. D. van Enter \\ Institute for Theoretical Physics, University of Groningen, \\ P.O. Box 800, Groningen, Netherlands
}

\begin{abstract}
In two recent papers Ruelle gave a heuristic theory of phase transitions, using some techniques introduced by Israel. He proves a version of the Gibbs phase rule, assuming a differentiability condition for the pressure. Ruelle already pointed out that his condition cannot always hold. In this paper we prove that the interaction spaces which he considers are in general too large for his condition to hold. We also show that the version of the Gibbs phase rule which is a consequence of this condition does not hold in general. Moreover we give some constraints on the analyticity properties of the pressure.
\end{abstract}

\section{Introduction}

In two recent papers Ruelle $[1,2]$ proposed a heuristic theory of phase transitions. He shows that every interaction which admits $n$ phases lies in a manifold of codimension $n-1$ of interactions which admit $n$ or more phases, if the pressure is differentiable in a certain sense.

In this paper we will study his differentiability condition. We will firstly show that on the usual space of interactions of which the pressure is defined, the condition never holds. In the second part of our paper we consider a smaller space of interactions where it is possible to discriminate between differentiability (and also analyticity) properties at low and high temperatures. We will prove that in a more phase region the pressure is not Fréchet differentiable and therefore not analytic in the space of pair interactions (Theorem 1) (for 1-dimensional systems this result was proven by Ruelle [2]). Moreover we show that, in general, spaces of the type considered by Ruelle are too large to obtain manifolds of more phases and that the version of the Gibbs phase rule as proposed in [2] cannot be true (Theorem 2).

We follow Israel considering a quantum lattice (the same results hold for classical lattices).

I. We consider a lattice $\mathbb{Z}^{v}$. At each point $x \in \mathbb{Z}^{v}$, there is defined an identical $m$-dimensional Hilbert space $\mathfrak{H}_{x}$. For each finite subset $X$ of $\mathbb{Z}^{v}$ the Hilbert space $\mathfrak{H}_{X}=\bigotimes_{x \in X} \mathfrak{Y}_{x}$ is defined. 
II. The quasilocal $C^{*}$-algebra $\mathfrak{A}$ of observables is the $C^{*}$-inductive limit of the local algebras $\mathfrak{U}_{X}$ of all bounded linear operators on $\mathfrak{H}_{X}$. The translations $\tau_{x}, x \in \mathbb{Z}^{v}$ act as automorphisms on $\mathfrak{A}$. The translation invariant states are denoted $\mathrm{I}$.

III. An interaction $\Phi$ is a function on the finite subsets $X$ of $\mathbb{Z}^{v}$ to selfadjoint operators $\Phi(X) \in \mathfrak{U}_{X}$ with:

a) $\Phi(x+X)=\tau_{x}(\Phi(X))$

b) $\|\Phi\|=\sum_{0 \in X} N(X)^{-1}\|\Phi(X)\|<\infty$

where $N(X)$ is the number of points in $X$. With this norm the interactions form a separable Banach space $\mathscr{B}$. The observable $A_{\Phi}$ is defined by

$$
A_{\Phi}=\sum_{0 \in X} N(X)^{-1} \Phi(X) \in \mathfrak{A} .
$$

IV. The pressure $P$ is a convex function on $\mathscr{B}$ with

$$
|P(\Phi)-P(\Psi)| \leqq\|\Phi-\Psi\| \quad \text { for all } \quad \Phi, \Psi \in \mathscr{B} \text {. }
$$

V. The mean entropy $s$ is an affine upper semicontinuous (in the weak*topology) function on I.

VI. $P(\Phi)=\sup _{\varrho \in I}\left\{s(\varrho)-\varrho\left(A_{\Phi}\right)\right\}$; if $P(\Phi)=s(\varrho)-\varrho\left(A_{\Phi}\right)$ then $\varrho$ is called an invariant equilibrium state for $\Phi$.

VII. $s(\varrho)=\inf _{\Phi \in \mathscr{B}}\left\{P(\Phi)+\varrho\left(A_{\Phi}\right)\right\}$. There is a $1-1$ correspondence between linear functionals on $\mathscr{B}$ tangent to $P$ at $\Phi$, denoted $\alpha$, and invariant equilibrium states for $\Phi$, denoted $\varrho$, given by

$$
\alpha(\Psi)=-\varrho\left(A_{\Psi}\right) \text { for all } \Psi \in \mathscr{B} .
$$

VIII. Every translation invariant state has a unique decomposition in extremal invariant states. If $\varrho$ is an equilibrium state for $\Phi$, the decomposition is in extremal invariant equilibrium states for $\Phi$. We denote the set of invariant equilibrium states for $\Phi: I_{\Phi}$ and the set of extremal points of $I_{\Phi}: \operatorname{Ext}\left(I_{\Phi}\right)$.

We will use the following two notions of differentiability on Banach space $[4 \mathrm{a}, \mathrm{b}]$ :

\section{a) Differentiability in the Sense of Gâteaux}

A function $f$ from a Banach space $\mathscr{E}$ to a Banach space $\mathscr{F}$ is differentiable in the sense of Gâteaux at the point $x_{0}$, if there exists a linear operator $f^{\prime}\left(x_{0}\right): \mathscr{E} \rightarrow \mathscr{F}$ such that

$$
\lim _{\lambda \rightarrow 0} \frac{\left\|f\left(x_{0}+\lambda h\right)-f\left(x_{0}\right)-\lambda f^{\prime}\left(x_{0}\right) h\right\|}{\lambda}=0 \text { for all } h \in \mathscr{E} .
$$

\section{b) Differentiability in the Sense of Fréchet}

A function $f$ from a Banach space $\mathscr{E}$ to a Banach space $\mathscr{F}$ is differentiable in the sense of Fréchet at the point $x_{0}$, if there exists a linear operator $f^{\prime}\left(x_{0}\right): \mathscr{E} \rightarrow \mathscr{F}$ such 
that

$$
\lim _{\|h\| \rightarrow 0} \frac{\left\|f\left(x_{0}+h\right)-f\left(x_{0}\right)-f^{\prime}\left(x_{0}\right) h\right\|}{\|h\|}=0 .
$$

Clearly every Fréchet differentiable function is also Gâteaux differentiable. The converse is not true. A well-known example is the following [4b]:

Consider the Banach space $C([0,1])$ consisting of real continuous functions on $[0,1]$ with the supremum norm $\|\cdot\|_{\infty}$. The map $g \rightarrow\|g\|_{\infty}$ is convex and it is Gâteaux differentiable at each function with a unique absolute maximum of $|g|$. If $y_{0}$ is the unique maximum point of $|g|$, then the Gâteaux derivative is the linear map from $C([0,1])$ into $\mathbb{R}$ given by:

$$
h \rightarrow h\left(y_{0}\right) \quad \text { if } \quad g\left(y_{0}\right) \geqq 0
$$

or

$$
h \rightarrow-h\left(y_{0}\right) \text { if } g\left(y_{0}\right)<0 .
$$

The map $g \rightarrow\|g\|_{\infty}$ is nowhere Fréchet differentiable.

If $P$ is a convex function from a Banach space into $\mathbb{R}$, then Gâteaux differentiability is equivalent to having a unique tangent plane [4b].

\section{Differentiability on the Space $\mathscr{B}$}

We will first give two lemmas, which will be used in the proof of Proposition (1). The first lemma is an immediate consequence of VII.

Lemma 1. Let $\Phi_{k} \in \mathscr{B}$, for $k \in \mathbb{N}$, such that $\lim _{k \rightarrow \infty} \Phi_{k}=\Phi$. Suppose $\varrho_{k} \in I_{\Phi_{k}}$ and $\varrho \in I_{\Phi}$ satisfy $w_{k \rightarrow \infty}^{*}-\lim _{k} \varrho_{k}=\varrho$. Then

$$
\lim _{k \rightarrow \infty} s\left(\varrho_{k}\right)=s(\varrho)
$$

Proof. Using the variational principle VII we have

$$
s(\varrho)=P(\Phi)-\varrho\left(A_{\Phi}\right)
$$

and

$$
s\left(\varrho_{k}\right)=P\left(\Phi_{k}\right)-\varrho_{k}\left(A_{\Phi_{k}}\right) .
$$

Hence

$$
\begin{aligned}
\left|s\left(\varrho_{k}\right)-s(\varrho)\right| & \leqq\left|P\left(\Phi_{k}\right)-P(\Phi)\right|+\left|\varrho_{k}\left(A_{\Phi_{k}}\right)-\varrho_{k}\left(A_{\Phi}\right)\right|+\left|\varrho_{k}\left(A_{\Phi}\right)-\varrho\left(A_{\Phi}\right)\right| \\
& \leqq 2 \cdot\left\|\Phi_{k}-\Phi\right\|+\left|\left(\varrho_{k}-\varrho\right)\left(A_{\Phi}\right)\right| .
\end{aligned}
$$


Therefore

$\lim _{k \rightarrow \infty} s\left(\varrho_{k}\right)=s(\varrho)$.

The second lemma follows from a result in [3].

Lemma 2. Let $\Phi \in \mathscr{B}$ such that $n+1$ pure phases coexist for $\Phi$ which will be denoted by $\sigma_{0}, \ldots, \sigma_{n}$. So $I_{\Phi}$ is a $n$-dimensional simplex and $\operatorname{Ext}\left(I_{\Phi}\right)=\left\{\sigma_{0}, \ldots, \sigma_{n}\right\}$. Then there exists a sequence of interactions $\left\{\Phi_{k}\right\} \subset \mathscr{B}$ and a sequence of states $\left\{\varrho_{k}\right\} \subset I$ such that

(i) $\lim _{k \rightarrow \infty} \Phi_{k}=\Phi$.

(ii) $\varrho_{k}$ is an extremal invariant equilibrium state for $\Phi_{k}$.

(iii) $w_{k \rightarrow \infty}^{*} \lim _{k}=\varrho$ and $\varrho \in I_{\Phi}$.

(iv) $\left\|\varrho_{k}-\varrho\right\|=2$ for all $\mathrm{k}$.

Proof. It follows from Theorem $3^{c}$ in [3] that there exists a sequence $\left\{\Psi_{\ell}\right\} \subset \mathscr{B}$ with $\lim _{\ell \rightarrow \infty} \Psi_{\ell}=\Phi$ and for each $\Psi_{\ell}$ there exist uncountably many ergodic equilibrium states. Because $I_{\Phi}$ has only $n+1$ extremal points we can choose for each $\ell$ a state $\varrho_{\ell}^{\prime} \in \operatorname{Ext}\left(I_{\Psi_{\ell}}\right)$, such that $\varrho_{\ell}^{\prime} \notin I_{\Phi}$. Since the unit ball in $\mathfrak{A}^{*}$ is $w^{*}$-compact there exists a $w^{*}$-convergent subsequence $\left\{\varrho_{k}\right\}$, each $\varrho_{k}$ equilibrium state for some $\Psi_{\ell}$, which will be called $\Phi_{k}$, such that $w_{k \rightarrow \infty}^{*}-\lim \varrho_{k}=\varrho$. (Note that we can deal with a subsequence instead of a subnet since the unit ball of $\mathfrak{I}^{*}$ is metrizable, because $\mathfrak{U}$ itself is separable.) Let $\alpha_{k}$ denote the tangent to $P$ at $\Phi_{k}$ corresponding to the state $\varrho_{k}$ then :

$$
\forall \Psi \in \mathscr{B}: P\left(\Phi_{k}+\Psi\right) \geqq P\left(\Phi_{k}\right)+\alpha_{k}(\Psi) .
$$

Since $w^{*}-\lim \varrho_{k}=\varrho$ we also have $w^{*}-\lim \alpha_{k}=: \alpha$ defines a linear functional on $\mathscr{B}$. Taking the limit $k \rightarrow \infty$ at both sides of (1) we obtain

$$
\forall \Psi \in \mathscr{B}: P(\Phi+\Psi) \geqq P(\Phi)+\alpha(\Psi)
$$

hence $\alpha$ is a tangent to $P$ at $\Phi$, and therefore $\varrho \in I_{\Phi}$.

The only thing left to prove is (iv). It follows from [3] that $\left\|\varrho_{k}-\varrho\right\|=\left\|\mu_{k}-\mu\right\|$, where $\mu_{k}$ and $\mu$ denote the measures on $I$ with barycenter $\varrho_{k}$ respectively $\varrho$. Obviously $\mu_{k}$ is a $\delta$-measure concentrated at the point $\varrho_{k}$. The measure $\mu$ is concentrated at the extremal points of $I_{\Phi}$. Since $\varrho_{k} \notin I_{\Phi}$ we conclude

$$
\left\|\mu_{k}-\mu\right\|=2
$$

and therefore

$$
\left\|\varrho_{k}-\varrho\right\|=2 .
$$

Remark. In most cases of physical interest the result follows more directly, without using Theorem $3^{c}$ in [3]. It has been proved by Griffiths and Ruelle in [5a], for classical systems, that the pressure is strictly convex on the space of interactions with $\|\Phi\|=\sum_{0 \in X}|\Phi(X)|<\infty$. In [5b] Roos proves the same result in the quantum case, for the space of interactions with $\|\Phi\|=\sum_{0 \in X}\|\Phi(X)\| e^{N(X)}<\infty$. In both cases we could simplify the proof of Lemma 2 by taking for $\Phi_{k}$ interactions at different 
temperature. So $\Phi_{k}=\beta_{k}$. $\Phi$ with $\beta_{k} \rightarrow 1$, and the $\varrho_{k}$ 's extremal invariant equilibrium states for $\Phi_{k}$. It follows then from the strict convexity of the pressure that $\varrho_{k} \notin I_{\Phi}$.

In the following proposition we will prove that the pressure is nowhere Fréchet differentiable.

Proposition 1. The pressure $P$ is not Fréchet differentiable at any $\Phi \in \mathscr{B}$.

Proof. It is clear that we may restrict ourselves to $\Phi \in \mathscr{B}$ for which the pressure has a unique tangent at $\Phi$, otherwise the pressure is not even Gâteaux differentiable. So $I_{\Phi}$ consists of a single state say $\varrho$. The Gâteaux derivative at $\Phi$ is the linear map $\Psi \rightarrow-\varrho\left(A_{\Psi}\right)$. We now use Lemma 2 (for the special case $n=0$ ) to ensure the existence of sequences $\left\{\Phi_{k}\right\}$ and $\left\{\varrho_{k}\right\}$ with the properties (i) to (iv). It follows from [3] that $\left\|\alpha_{k}-\alpha\right\|=\left\|\varrho_{k}-\varrho\right\|=2$, where $\alpha_{k}$ and $\alpha$ are the tangents to the pressure at $\Phi_{k}$ resp. $\Phi$ corresponding to $\varrho_{k}$ and $\varrho$. Hence:

$$
\sup _{\|\Psi\|=1}\left|\left(\varrho_{k}-\varrho\right)\left(A_{\Psi}\right)\right|=\sup _{\|\Psi\|=1}\left|\left(\alpha_{k}-\alpha\right) \Psi\right|=\left\|\alpha_{k}-\alpha\right\|=2 .
$$

Choose $\delta>0$ arbitrary. Using Lemma 1 and Lemma 2 (iii) we have

$$
\left|s\left(\varrho_{k}\right)-s(\varrho)\right|<\frac{1}{2} \delta
$$

and

$$
\left|\left(\varrho_{k}-\varrho\right)\left(A_{\Phi}\right)\right|<\frac{1}{2} \delta
$$

if $k$ is sufficiently large. Note that VI implies

$$
P(\Phi+\Psi) \geqq s\left(\varrho_{k}\right)-\varrho_{k}\left(A_{\Phi+\Psi}\right)
$$

and therefore

$$
P(\Phi+\Psi)-P(\Phi)+\varrho\left(A_{\Psi}\right) \geqq s\left(\varrho_{k}\right)-s(\varrho)+\left(\varrho-\varrho_{k}\right)\left(A_{\Phi}\right)+\left(\varrho-\varrho_{k}\right)\left(A_{\Psi}\right) .
$$

Using (3) and (4) we obtain:

$$
P(\Phi+\Psi)-P(\Phi)+\varrho\left(A_{\Psi}\right) \geqq\left(\varrho-\varrho_{k}\right)\left(A_{\Psi}\right)-\delta .
$$

It follows from (2) that

$$
\sup _{\|\Psi\|=\delta} \frac{\left|P(\Phi+\Psi)-P(\Phi)+\varrho\left(A_{\Psi}\right)\right|}{\|\Psi\|} \geqq 1 .
$$

Since $\delta$ was arbitrary, $P$ cannot be Fréchet differentiable at $\Phi$.

Let us now clarify the relevance of the notion of Fréchet differentiability for the Gibbs phase rule, by citing the following theorem of Ruelle [2]. Let $\mathscr{B}_{0}$ be a subspace of $\mathscr{B}$ with norm $\|\cdot\|_{0}$.

Theorem. Let $\Phi \in \mathscr{B}$ and let $I_{\Phi}$ be an $n$-dimensional simplex with $\operatorname{Ext}\left(I_{\Phi}\right)$ $=\left\{\sigma_{0}, \ldots, \sigma_{n}\right\}$. Let $\beta_{0}, \ldots, \beta_{n}$ denote the corresponding tangents. Suppose that $\beta_{1}-\beta_{0}, \beta_{2}-\beta_{0}, \ldots, \beta_{n}-\beta_{0}$ are linearly independent and let $\mathscr{X}$ be the linear space: $\mathscr{X}=\left\{\Psi \in \mathscr{B}_{0} \mid \beta_{0}(\Psi)=\ldots=\beta_{n}(\Psi)\right\}$. $\mathscr{X}$ is of codimension $n$. Assume that

$(R)$ : The restriction of $P$ to the linear manifold $\Phi+\mathscr{X}$ is Fréchet differentiable at $\Phi$. 
Suppose furthermore that there is a n-dimensional subspace $\mathscr{Y}$ of $\mathscr{B}_{0}$ such that $\mathscr{X} \oplus \mathscr{Y}=\mathscr{B}_{0}$ and $\lambda \geqq 0$ such that if $\Omega \in \mathscr{Y}$ and $x \in \mathbb{Z}^{v}$ there exists $\Phi_{x} \in \mathscr{B}_{0}$ with:

$$
\begin{aligned}
& \varrho\left(A_{\Omega} \cdot A_{\Omega} \circ \tau^{x}\right)=\varrho\left(A_{\Phi_{x}}\right) \quad \text { for all } \varrho \in I \\
& \left\|\Phi_{x}\right\|_{0} \leqq \lambda \cdot\|\Omega\|_{0}^{2} .
\end{aligned}
$$

For every non-empty subset $K=\left\{i_{0}, \ldots, i_{k}\right\}$ of $\{0,1, \ldots, n\}$, let

$$
\mathscr{X}_{k}=\left\{\Psi \in \mathscr{B}_{0} \mid \beta_{i_{0}}(\Psi)=\ldots=\beta_{i_{k}}(\Psi) \geqq \beta_{j}(\Psi), j \notin K\right\} .
$$

There is a homeomorphism $\boldsymbol{h}$ of a neighbourhood $\mathcal{O} \subset \mathscr{B}_{0}$ of $\Phi$ to a neighbourhood of $\Phi$ such that $\boldsymbol{h}$ is strictly differentiable at $\Phi$, with derivative the identity and for every $\Psi \in h\left(\left(\Phi+\mathscr{X}_{k}\right) \cap \mathcal{O}\right)$ at least $\boldsymbol{k}+1$ phases coexist.

On the basis of our proposition we will show that condition $(R)$ does not hold if $\mathscr{B}_{0}=\mathscr{B}$. Because all tangents to $P$ at $\Phi$ coincide on $\mathscr{X}$, the restriction of $P$ to the linear manifold $\Phi+\mathscr{X}$ is Gâteaux differentiable at $\Phi$ and the Gâteaux derivative is the map $\Psi \rightarrow-\sigma_{0}\left(A_{\Psi}\right)$. We will prove:

$$
\exists_{\varepsilon>0} \forall_{\delta>0} \sup _{\Psi \in \mathscr{X},\|\Psi\|=\delta} \frac{\left|P(\Phi+\Psi)-P(\Phi)+\sigma_{0}\left(A_{\Psi}\right)\right|}{\|\Psi\|} \geqq \varepsilon
$$

and therefore $P$ restricted to $\Phi+\mathscr{X}$ is not Fréchet differentiable at $\Phi$.

We use Lemma 2 again to guarantee the existence of sequences $\left\{\Phi_{k}\right\}$ and $\left\{\varrho_{k}\right\}$ with the properties (i) to (iv). As in the proposition we have:

$$
\sup _{\|\Psi\|=1}\left|\left(\varrho_{k}-\varrho\right)\left(A_{\Psi}\right)\right|=\sup _{\|\Psi\|=1}\left|\left(\alpha_{k}-\alpha\right) \Psi\right|=2
$$

where $\alpha_{k}$ and $\alpha$ are the tangents corresponding to $\varrho_{k}$ resp. $\varrho$.

Now let $P_{\mathscr{X}}$ and $P_{\mathscr{Y}}$ denote the projection operators on $\mathscr{X}$ respectively $\mathscr{Y}$ defined by the direct sum $\mathscr{X} \oplus \mathscr{Y}=\mathscr{B}$. It follows from the closed graph theorem [6] that both $P_{\mathscr{X}}$ and $P_{\mathscr{y}}$ are bounded. Since $\alpha_{k} \rightarrow \alpha$ pointwise on $\mathscr{B}$ and $\operatorname{dim} \mathscr{Y}=n$ we have $\alpha_{k} \circ P_{\mathscr{y}} \rightarrow \alpha \circ P_{\mathscr{y}}$ uniform on $\mathscr{B}$. Hence:

$$
\begin{aligned}
2=\sup _{\|\Psi\|=1}\left|\left(\alpha_{k}-\alpha\right) \Psi\right| & =\sup _{\|\Psi\|=1}\left|\left(\alpha_{k}-\alpha\right) \circ P_{\mathscr{X}} \Psi+\left(\alpha_{k}-\alpha\right) \circ P_{\mathscr{Y}} \Psi\right| \\
& \leqq \sup _{\|\Psi\|=1}\left|\left(\alpha_{k}-\alpha\right) \circ P_{\mathscr{X}} \Psi\right|+1
\end{aligned}
$$

if $k$ is sufficiently large. From this we easily deduce:

$$
\sup _{\Psi \in \mathscr{X},\|\Psi\|=1}\left|\left(\alpha_{k}-\alpha\right) \Psi\right| \geqq \frac{1}{\left\|P_{\mathscr{X}}\right\|} .
$$

Fix $\delta>0$ arbitrary. Using (6), Lemma 1 and Lemma 2 we can choose $k$ large enough to ensure that:

$$
\begin{aligned}
& \sup _{\Psi \in \mathscr{X},\|\Psi\|=\delta}\left|\left(\varrho_{k}-\varrho\right)\left(A_{\Psi}\right)\right| \geqq \frac{\delta}{\left\|P_{\mathscr{X}}\right\|} \\
& \left|s\left(\varrho_{k}\right)-s(\varrho)\right| \leqq \frac{\delta}{4\left\|P_{\mathscr{X}}\right\|} \\
& \left|\left(\varrho_{k}-\varrho\right)\left(A_{\Phi}\right)\right| \leqq \frac{\delta}{4\left\|P_{\mathscr{X}}\right\|} .
\end{aligned}
$$


Note furthermore that $\varrho$ coincides with $\sigma_{0}$ on observables of the form $A_{\Psi}$ with $\Psi \in \mathscr{X}$. Combining (5) with (7), (8), and (9) we obtain:

$$
\sup _{\Psi \in \mathscr{X},\|\Psi\|=\delta} \frac{\left|P(\Phi+\Psi)-P(\Phi)+\sigma_{0}\left(A_{\Psi}\right)\right|}{\|\Psi\|} \geqq \frac{1}{2\left\|P_{\mathscr{X}}\right\|}
$$

which completes the proof.

Israel has shown in [3] that the space of interactions considered is, from the physical point of view, too large. It might be conjectured that Ruelle's theorem holds in smaller spaces. In the next sections we will show however that even in smaller spaces, e.g. the space of pair interactions the pressure is not Fréchet differentiable in more phase regions.

\section{Fréchet Differentiability in Smaller Spaces}

Firstly we prove a lemma which will be used in the proof of Theorem 1.

Let $\varrho$ be a density matrix on a finite lattice $\Lambda \subset \mathbb{Z}^{v}, \varrho$ the corresponding state. Let $X \subset \mathbb{Z}^{v}$, define the operator $R_{X}$ by

$$
\begin{array}{lllll}
R_{X} A_{j}=D^{\left(\frac{m-1}{2}\right)^{+}}(\pi, \mathbf{r}) A_{j} D^{\left(\frac{m-1}{2}\right)}(\pi, \mathbf{r}) & \text { if } j \in X & \text { and } & A_{j} \in \mathfrak{U}_{j} \\
R_{X} A_{j}=A_{j} & \text { if } & j \notin X & \text { and } & A_{j} \in \mathfrak{U}_{j}
\end{array}
$$

where $D^{\left(\frac{m-1}{2}\right)}(\pi, \mathbf{r})$ is a unitary $m \times m$ matrix, such that $R_{X}$ gives rise to a rotation of magnitude $\pi$ around some axis $\mathbf{r}$ at each point $j \in X$. So $R_{X}$ rotates in particular the spins in the sublattice $X$ over an angle $\pi . R_{X}^{-1} \varrho \underline{Q}$ is the density matrix corresponding to $\varrho \circ R_{X}$.

Lemma 3. The entropy of the state $\varrho, S_{\varrho}(\Lambda)$, equals $S_{\varrho \circ R_{X}}(\Lambda)\left(=S_{\varrho \circ R_{X} \cap \Lambda}(\Lambda)\right)$.

Proof. $R_{X}^{-1}$ is unitarily implementable on $\mathfrak{H}(\Lambda)$ by the unitary matrix $U$.

$$
\begin{aligned}
S_{\varrho \circ R_{X}}(\Lambda) & =-\operatorname{Tr}_{\mathfrak{S}(\Lambda)}\left(R_{X}^{-1} \bar{\varrho} \ln R_{X}^{-1} \bar{\varrho}\right)=-\sum_{i=1}^{m^{N(\Lambda)}}\left(\phi_{i}, R_{X}^{-1} \bar{\varrho} \ln R_{X}^{-1} \bar{\varrho} \phi_{i}\right) \\
& =-\sum_{i=1}^{m^{N(\Lambda)}}\left(U \phi_{i}, \bar{\varrho} \ln \varrho U \phi_{i}\right)=-\operatorname{Tr}_{\mathfrak{S}(\Lambda)}(\bar{\varrho} \ln \varrho)=S_{\varrho}(\Lambda) .
\end{aligned}
$$

Without loss of generality we will further assume that $R_{X} s_{0}^{z}=-s_{0}^{z}$ if $0 \in \mathscr{X}$.

Definition 1. We will define a state to be a periodic equilibrium state for $\Phi$, if it appears in the decomposition at infinity [9] of some element of $\operatorname{Ext}\left(I_{\Phi}\right)$ and is invariant under some subgroup of the translations. We will denote the periodic equilibrium states for $\Phi: I_{\Phi}^{p}$ and the extremal periodic equilibrium states for $\Phi: \operatorname{Ext}\left(I_{\Phi}^{p}\right)$. Clearly $I_{\Phi} \subset I_{\Phi}^{p}$.

If some periodic state $\varrho$ of period $n_{1}, \ldots, n_{v}$ in the $v$ directions is an equilibrium state for $\Phi$ then the states $\varrho \circ \tau_{i_{j}}$ are equilibrium states for $\Phi$ for $i_{j}=1$ to $n_{j}, j=1 \ldots v$.

The state $\tilde{\varrho}=\frac{1}{n_{1} \ldots n_{v}} \sum_{i_{1}=1}^{n_{1}} \ldots \sum_{i_{v}=1}^{n_{v}} \varrho \circ \tau_{i_{1}, \ldots, i_{v}} \in I_{\Phi}$. We will from now on only consider periodicity in one direction. 
Definition 2. An interaction $\Psi$ does not break the symmetry at $\Phi$ if $\varrho\left(A_{\Psi}\right)$ has the same value for all $\varrho \in \operatorname{Ext}\left(I_{\Phi}\right)$. Note that if $\varrho_{1}, \varrho_{2} \in I_{\Phi}^{p}$ with period $n$ and $\Psi$ does not break the symmetry at $\Phi$ then

$$
\tilde{\varrho}_{1}\left(A_{\Psi}\right)=\tilde{\varrho}_{2}\left(A_{\Psi}\right) \text { where } \tilde{\varrho}_{i}=\frac{1}{n} \sum_{j=1}^{n} \varrho_{i} \tau_{j} .
$$

We will now consider mappings $R_{X}$ where $X$ is chosen such that $R_{X}$ is a symmetry of the system which leaves the interaction invariant. In many models (e.g. ferro- or antiferromagnetically ordered Ising or Heisenberg systems) $X=\mathbb{Z}^{v}$, in other models (e.g. the Baxter-Wu model) $X$ will be a periodic sublattice of $\mathbb{Z}^{v}$. We are interested in the case where this symmetry is broken. For simplicity we assume that $\operatorname{Ext}\left(I_{\Phi}\right)\left(\operatorname{Ext}\left(I_{\Phi}^{p}\right)\right)$ consists of 2 states (possibly together with their translates). However the following theorems can be generalized to all cases where the subspace of pair interactions, which do not break the symmetry, has infinite dimension.

Theorem 1. Let $\operatorname{Ext}\left(I_{\Phi}\right)\left(\operatorname{Ext}\left(I_{\Phi}^{p}\right)\right)=\left\{\varrho_{1}, \varrho_{2}\right\}$ (and possibly their translates). Let $R_{X}$ be such that $\varrho_{2}=\varrho_{1} \circ R_{X}$ and $\varrho_{2}\left(s_{0}^{z}\right) \neq \varrho_{1}\left(s_{0}^{z}\right)$. Then the pressure is not differentiable in the sense of Fréchet on the subspace of pair interactions which do not break the symmetry at $\Phi$.

Proof. Divide the lattice in layers $L_{N}^{i}$ of thickness $N$ in one direction

$$
\left(L_{N}^{i}=\left\{x=\left(x_{1} \ldots x_{v}\right) \mid x \in \mathbb{Z}^{v}, N i \leqq x_{1}<N(i+1)\right\}\right) .
$$

Assume that $\varrho_{1}$ and $\varrho_{2}$ have period $n$ in that direction. Then $X$ has a period which is at most $n$, for if $\varrho_{1}\left(R_{X} s_{0}^{z}\right) \neq \varrho_{1}\left(s_{0}^{z}\right)$ i.e. $0 \in X$ then $\varrho_{1}\left(R_{X} \tau_{n k} s_{0}^{z}\right) \neq \varrho_{1}\left(\tau_{n k} s_{0}^{z}\right)$ i.e. $\tau_{n k} 0 \in X \forall k$.

Define operators $R_{N}$ by

$$
R_{N}=R_{Y} \quad \text { with } \quad Y=X \cap\left(\bigcup_{i} L_{N}^{2 i+1}\right) .
$$

So $R_{N}$ rotates part of the spins in half of the layers (if $X=\mathbb{Z}^{v}$ all the spins in half of the layers are rotated).

We define $\tilde{\varrho}_{N n}$ by

$$
\tilde{\varrho}_{N n}(A)=\frac{1}{2 N n} \sum_{i=1}^{2 N n} \varrho_{1}\left(R_{N n} \tau_{i} A\right)\left(=\frac{1}{2 N n} \sum_{i=1}^{2 N n} \varrho_{2}\left(R_{N n} \tau_{i} A\right)\right) .
$$

Note that $\tilde{\varrho}_{N n}$ is translation invariant (by construction) and $s\left(\tilde{\varrho}_{N n}\right)=s\left(\varrho_{1}\right)$ by Lemma 3 and the fact that $s$ is affine [12, Chap. 7].

Let $\Psi$ be a finite range interaction (so $A_{\Psi}$ is local), which does not break the symmetry at $\Phi$. Then

$$
\begin{aligned}
& \left|\tilde{\varrho}_{N n}\left(A_{\Psi}\right)-\tilde{\varrho}_{1}\left(A_{\Psi}\right)\right|=\frac{1}{2 N n}\left|\sum_{i=1}^{2 N n} \varrho_{1}\left(\left(R_{N n}-1\right) \tau_{i} A_{\Psi}\right)\right| \leqq \frac{2 \operatorname{range}(\Psi)}{N n}\|\Psi\| \\
& \left(\tilde{\varrho}_{1}(A)=\frac{1}{n} \sum_{i=1}^{n} \varrho_{1}\left(\tau_{i} A\right) \quad \text { so } \quad \tilde{\varrho}_{1} \in I_{\Phi}\right) .
\end{aligned}
$$


(Only those $\tau_{i} A_{\Psi}$ contribute to the sum that have support in two neighbouring layers, for $N$ large enough, the other terms vanish because $\frac{1}{n} \sum_{i=1}^{n} \varrho_{1}\left(R_{X} \tau_{i} A_{\Psi}\right)$ $=\frac{1}{n} \sum_{i=1}^{n} \varrho_{1}\left(\tau_{i} A_{\Psi}\right)(\Psi$ does not break the symmetry at $\Phi)$ and hence, if $\sum_{j=1}^{n} \tau_{i+j} A_{\Psi}$ has support in one layer $\sum_{j=1}^{n} \varrho_{1}\left(R_{N} \tau_{i+j} A_{\Psi}\right)=\sum_{i=1}^{n} \varrho_{1}\left(\tau_{i+j} A_{\Psi}\right)$.)

So if $\mathfrak{U}^{0}$ is the linear subspace of $\mathfrak{A}$ on which the restriction of both $\tilde{\varrho}_{1}$ and $\tilde{\varrho}_{2}$ coincide $\tilde{\varrho}_{N n}$ converges to $\tilde{\varrho}_{1}$ on the local elements of $\mathfrak{U}^{0}$ and hence on $\mathfrak{U}^{0}$ pointwise if $N$ tends to infinity.

We now consider the product of two spins in the $z$ direction at a distance which is equal to the thickness of the layers.

Let

$$
\lim _{N \rightarrow \infty} \tilde{\varrho}_{1,2}\left(s_{0}^{z} \tau_{N n} s_{0}^{z}\right)=m_{1}>0
$$

where $N$ runs through some subsequence of $\mathbb{N}$ (this follows from the weak cluster property which is consequence of extremal invariance [12, Chap. 6;15]).

Then

$$
\lim _{N \rightarrow \infty} \tilde{\varrho}_{N n}\left(s_{0}^{z} \tau_{N n} s_{0}^{z}\right)=m_{2} \leqq m_{1}\left(1-\frac{1}{n}\right) \varsubsetneqq m_{1}
$$

by noticing that inside a layer a fixed fraction of at least $\frac{1}{n}$ of the spins is rotated.

Let $\Psi_{N}$ be the $R_{X}$-invariant pair interaction between spins with distance $N$ and strength 1 such that $A_{\Psi_{N}}=\frac{1}{2}\left(s_{0}^{z} \tau_{N} s_{0}^{z}+s_{0}^{z} \tau_{-N} s_{0}^{z}\right)$. Note that the norm of $\Psi_{N}$ does not depend on $N$. Now

$$
\begin{gathered}
P\left(\Phi+\varepsilon \Psi_{N}\right)-P(\Phi)+\varepsilon \tilde{\varrho}_{1}\left(A_{\Psi_{N}}\right) \geqq s\left(\tilde{\varrho}_{N}\right)-\tilde{\varrho}_{N}\left(A_{\Phi}\right)-\varepsilon \tilde{\varrho}_{N}\left(A_{\Psi_{N}}\right)-s\left(\tilde{\varrho}_{1}\right) \\
+\tilde{\varrho}_{1}\left(A_{\Phi}\right)+\varepsilon \tilde{\varrho}_{1}\left(A_{\Psi_{N}}\right) .
\end{gathered}
$$

So if $N=N_{1} n$ sufficiently large, by $(10),(12)$ and the fact that $s\left(\tilde{\varrho}_{N}\right)=s\left(\tilde{\varrho}_{1}\right)$ we have

$$
\frac{P\left(\Phi+\varepsilon \Psi_{N}\right)-P(\Phi)+\varepsilon \tilde{\varrho}_{1}\left(A_{\Psi_{N}}\right)}{\varepsilon} \geqq \frac{1}{2}\left(m_{1}-m_{2}\right) .
$$

and therefore $P$ is not Fréchet differentiable.

Theorem 2. Let $\Phi$ be as in Theorem 1. Then there exists for all $\varepsilon>0$ and all $N \in \mathbb{N}$ some $\xi$ in the space of pair interactions such that $\|\xi\|<\varepsilon$ and in the decomposition at infinity of some element of $\operatorname{Ext}\left(I_{\Phi+\xi}\right)$ there exist at least $N$ more periodic states than in the decomposition of any element of $\operatorname{Ext}\left(I_{\Phi}\right)$.

Proof. We consider again $\tilde{\varrho}_{N}$ as in Theorem 1. It follows from a theorem of Israel [3] (see also Ruelle $[1,13]$ ) that there exists a pair interaction $\xi$ with $\varrho_{\Phi+\xi} \in I_{\Phi+\xi}$ such that

$$
\sup _{\substack{\Psi \text { pairinteraction } \\\|\Psi\| \|=1}}\left|\varrho_{\Phi+\xi}\left(A_{\Psi}\right)-\tilde{\varrho}_{N}\left(A_{\Psi}\right)\right| \leqq \sqrt{\varepsilon}
$$


and

$$
\|\xi\| \leqq \frac{1}{\sqrt{\varepsilon}}\left(P(\Phi)-s\left(\tilde{\varrho}_{N}\right)+\tilde{\varrho}_{N}\left(A_{\Phi}\right)\right) \leqq \sqrt{\varepsilon}
$$

for $N=N_{1} n$ large enough.

We now choose $\sqrt{\varepsilon}<\frac{1}{2}\left(m_{1}-m_{2}\right)$.

Note that $\tilde{\varrho}_{N}$ has, by construction, a non trivial decomposition into extremal periodic states of period $2 \mathrm{~N}$ and

$$
\lim _{k \rightarrow \infty} \frac{1}{k} \sum_{i=1}^{k} \tilde{\varrho}_{N}\left(s_{0}^{z} \tau_{i \cdot 2 N} s_{0}^{z}\right) \neq\left(\tilde{\varrho}_{N}\left(s_{0}^{z}\right)\right)^{2} .
$$

Since by (14) $\varrho_{\Phi+\xi}$ and $\tilde{\varrho}_{N}$ are almost equal on a suitable set of $A_{\Psi}$, we have also

$$
\lim _{k \rightarrow \infty} \frac{1}{k} \sum_{i=1}^{k} \varrho_{\Phi+\xi}\left(s_{0}^{z} \tau_{i \cdot 2 N} s_{0}^{z}\right) \neq\left(\varrho_{\Phi+\xi}\left(s_{0}^{z}\right)\right)^{2}
$$

if $\varepsilon$ is small enough, and therefore $\varrho_{\Phi+\xi} \notin \operatorname{Ext}\left(I_{\Phi}^{2 N}\right)$. Hence $\varrho_{\Phi+\xi}$ has a decomposition in at least $2 \mathrm{~N}$ periodic states.

Examples where our theorems hold are all even classical interactions with ferro- or antiferromagnetic ordering, all systems where the rotation symmetry of the interaction is broken and with an obvious modification, the Baxter-Wu model.

\section{Constraints on Analyticity Properties}

The following theorem of Hille and Phillips makes it possible to prove constraints on analyticity properties [7].

Theorem. If a function $f$ is analytic in some open domain (which means analytic in all directions, locally bounded and Gâteaux differentiable) then $f$ is Fréchet differentiable in that domain. Moreover the Taylor expansion has a finite convergence radius for all points in the domain.

It follows directly from Theorem 1 that at all interactions which satisfy the conditions of Theorem 1 the pressure is not analytic in the space of pair interactions which do not break the symmetry. Let $g$ be a positive function on the subsets $X \subset \mathbb{Z}^{v}$ such that $\mathscr{B}_{g}$ is the corresponding subspace of $\mathscr{B}$ determined by

$$
\|\Psi\|_{g}=\sum_{0 \in X} g(X)\|\Psi(X)\|<\infty
$$

then in view of Theorem 1, the pressure, in the case of more phases, can be analytic at best in a subspace $\mathscr{B}_{g}$ where $g$ is a positive function which increases as the diameter of $X, D(X)$, increases for $N(X)=2$ (so the pair interactions in $\mathscr{B}_{g}$ form - a smaller - subspace of the interactions considered in Theorem 1). In fact one has to impose a stronger condition on $g$ to obtain analyticity as the following theorem shows. 

Theorem 3. If for $N(X)=2 \lim _{\operatorname{range}(X) \rightarrow \infty} \frac{g(X)}{\sqrt{D(X)}}=0$ and $\Phi$ is as in Theorem 1 then the
pressure is not analytic in $\mathscr{B}_{g}$.

Proof. If $P$ were analytic then $\exists C_{1}, C_{2}>0$ with

$$
P(\Phi+\Psi)-P(\Phi)+\varrho_{1}\left(A_{\Psi}\right) \leqq \frac{C_{1}\|\Psi\|_{g}^{2}}{C_{2}\left(C_{2}-2\|\Psi\|_{g}\right)}
$$

for all $\Psi$ with $\|\Psi\|_{g} \leqq \frac{1}{2} C_{2}$ (as follows from the uniform convergence of the Taylor expansion [7]).

Without loss of generality we will assume that the elements of $\mathscr{B}_{g}$ do not break the symmetry at $\Phi$ (if they do the theorem is obvious).

We will give the proof for $\Phi$ finite range, the general case can be proven with a little more effort. We will use the notation of Theorem 1. Let $\Psi_{N}$ be the pair interaction with strength 1 and range $N$. Define $g^{\prime}(N):=\left\|\Psi_{N}\right\|_{g}$.

From (13) we have

$$
P\left(\Phi+\varepsilon \Psi_{N}\right)-P(\Phi)+\varrho_{1}\left(A_{\varepsilon} \Psi_{N}\right) \geqq\left(m_{1}-m_{2}\right) \varepsilon-2 \frac{\operatorname{range}(\Phi)}{N}\|\Phi\| .
$$

We choose $\varepsilon_{N}$ dependent on $N$

$$
\varepsilon_{N}=\frac{4\|\Phi\|(\operatorname{range}(\Phi))}{\left(m_{1}-m_{2}\right) N}
$$

then for $N$ large enough and $C_{1}, C_{2}, m_{1}, m_{2}$ fixed

$$
\varepsilon_{N} g^{\prime}(N)^{2} \leqq \frac{C_{2}^{2}}{4 C_{1}}\left(m_{1}-m_{2}\right)
$$

and with (17) and (18):

$$
\begin{aligned}
& P\left(\Phi+\varepsilon_{N} \Psi_{N}\right)-P(\Phi)+\tilde{\varrho}_{1}\left(A_{\varepsilon_{N} \Psi_{N}}\right) \geqq \frac{1}{2}\left(m_{1}-m_{2}\right) \varepsilon_{N} \\
& \geqq 2 \frac{C_{1}}{C_{2}^{2}} \varepsilon_{N}^{2} g^{\prime}(N)^{2}=2 \frac{C_{1}}{C_{2}^{2}}\left\|\varepsilon_{N} \Psi_{N}\right\|_{g}^{2} .
\end{aligned}
$$

Now (19) contradicts (16).

On the other hand it is known that at low densities or high temperatures the pressure is analytic in a space $\mathscr{B}_{g}$ where $g$ is only dependent on the number of points in $X[8,10,14]$.

For classical systems Iagolnitzer and Souillard proved some theorems giving the equivalence between analyticity properties and strong cluster properties of the correlation functions [11].

They conjectured that it might be possible by analytic continuation to conserve the same analyticity and cluster properties in a pure phase below the critical temperature on a given side of the phase transition as in the low density region, provided one does not have symmetry breaking interactions. Our Theorem 3 shows that this is not true but that there are less analyticity properties in the low temperature region than in the high temperature region. 
Acknowledgements. We want to thank N. M. Hugenholtz and M. Winnink for helpful discussions and for the critical reading of the manuscript. This paper is part of the research program of the "Foundation for Fundamental Research on Matter" (F.O.M.), which is financially supported by the "Netherlands Organization for Pure Research" (Z.W.O.).

\section{References}

1. Ruelle, D.: On manifolds of phase coexistence. Theoret. Math. Phys. 30, $24-29$ (1977)

2. Ruelle, D.: A heuristic theory of phase transitions. Commun. Math. Phys. 53, 195-208 (1977)

3. Israel, R.B.: Existence of phase transitions for long-range interactions. Commun. Math. Phys. 43, 59-68 (1975)

4a. Vilenkin, N.: Functional analysis. Chapt. 4, Sects. 1, 2; Groningen: Wolters Noordhof 1972

4b. Roberts, A.M., Varberg, D.E.: Convex functions. Chapt. IV, Sect. 44; London, New York: Academic Press 1973

5a. Griffiths, R.B., Ruelle, D.: Strict convexity of the pressure on lattice systems. Commun. Math. Phys. 23, 169-175 (1971)

5b. Roos, H.: Strict convexity of the pressure. Commun. Math. Phys. 36, 263-276 (1974)

6. Dunford, N., Schwartz, J.T.: Linear operators I, Chapt. II, Sect. 2. New York: Wiley Interscience 1958

7. Hille, E., Phillips, R.: Functional analysis and semigroups. Chapt. III, Sect. 17; Baltimore: Waverly Press 1959

8. Gallavotti, G., Miracle-Sole, S. : Correlation functions of a lattice system. Commun. Math. Phys. 7, 274-288 (1967)

9. Lanford, O.E., Ruelle, D.: Observables at infinity and states with short range correlations in statistical mechanics. Commun. Math. Phys. 13, 194-215 (1969)

10. Greenberg, W.: Critical temperature bounds of quantum lattice gases. Commun. Math. Phys. 13, 335-344 (1969)

11. Iagolnitzer, D., Souillard, B.: On the analyticity in the potential in classical statistical mechanics. Commun. Math. Phys. 60, 131-152 (1978)

12. Ruelle, D.: Statistical mechanics. New York, Amsterdam: Benjamin 1969

13. Ruelle, D.: Thermodynamic formalism, Chapt. 3. Reading, Mass. : Addison-Wesley 1978

14. Gallavotti, G., Miracle-Sole, S., Robinson, D.W.: Phys. Lett. 25 A, 493-494 (1967)

15. Kastler, D., Robinson, D.W.: Commun. Math. Phys. 3, 151-180 (1966)

Communicated by E. Lieb

Received August 5, 1979 